\title{
DGHO:
}

\section{DRG in der Hämatologie und Internistischen Onkologie: Ergebnisse aus zwei deutschlandweiten Evaluationsprojekten}

\author{
S. W. Krause ${ }^{a}$ \\ M. Krych ${ }^{\mathrm{b}}$ \\ S. Glocker ${ }^{\mathrm{c}}$ \\ D. Franz ${ }^{d}$ \\ H. Ostermann ${ }^{b}$ \\ A. Ganser \\ N. Roeder ${ }^{\text {d }}$ \\ a Abteilung Hämatologie u. Internistische Onkologie, Klinikum der Universität Regensburg, \\ ${ }^{b}$ Medizinische Klinik und Poliklinik III, Klinikum Grosshadern, Universität München, \\ ${ }^{c}$ Abteilung für Radioonkologie mit Poliklinik, Radioonkologische Klinik, Universitätsklinikum Tübingen, \\ ${ }^{\mathrm{d}}$ DRG-Research-Group, Universitätsklinikum Münster, Germany
}

\section{Einleitung}

Seit dem Jahr 2000 müssen sich die Krankenhäuser darauf einstellen, dass die Vergütung für die stationäre Versorgung nach einem System von Fallpauschalen auf der Basis von Fallgruppen («Diagnosis Related Groups», DRG) abgerechnet wird. Als Basis für das deutsche Fallpauschalensystem wurde das australische AR-DRG-System gewählt, welches im Sinne eines «lernenden Systems» für die deutschen Bedürfnisse jährlich angepasst werden soll. Nach diesem System werden Gruppen von Behandlungsfällen einheitlich vergütet. Diese einheitliche Vergütung ist unabhängig davon, welches Krankenhaus die Leistung erbringt («gleiches Geld für gleiche Leistung»). Das Entgeltsystem soll nach der aktuellen Gesetzeslage nach Durchlaufen einer «Konvergenzphase» ab 2009 voll ökonomisch wirksam werden.

Es liegt in der Natur einer Vergütung über Fallgruppen, dass nicht der individuelle Patient aufwandsgerecht vergütet wird. Einzelne Patienten innerhalb einer Fallgruppe verursachen einen hohen und andere einen niedrigen Aufwand. Das DRGSystem kann trotzdem als «gerecht» akzeptiert werden, wenn die Homogenität der Fallgruppen gewährleistet ist und die einzelnen Fallgruppen bei allen Leistungserbringern eine vom finanziellen Aufwand her vergleichbare Mischung von Fällen enthalten. Einzelne «Ausreißer» begründen dementsprechend keine grundsätzliche Kritik am System.

Wenn sich innerhalb der Fallgruppen jedoch systematische Inhomogenitäten verbergen, so können diese zu einer Schieflage in der Vergütung führen. Inhomogenitäten innerhalb einzelner DRG sind insbesondere dann kritisch, wenn unterschiedlich aufwändige Patientenkollektive aufgrund unterschied- licher medizinische Schwerpunktbildung in verschiedenen Häusern behandelt werden, d. h. innerhalb einer DRG die «teuren» Fälle in Haus A und die «billigen» in Haus B.

\section{Anpassungsbedarf in der Onkologie}

Das DRG-System in Deutschland sieht ausdrücklich vor, dass Verbände und Fachgesellschaften der InEK GmbH («Institut für das Entgeltsystem im Krankenhaus», Institution der Selbstverwaltung) Anpassungsvorschläge für das DRG-System vorlegen. Dies soll zu einer Verbesserung des Systems beitragen. Die Vorschläge sollen konstruktive Lösungen im DRG System suchen und möglichst mit konkreten Zahlen von Behandlungsfällen belegt sein.

Den deutschen Onkologen wurde rasch klar, dass innerhalb ihres Fachgebiets erhebliche Inhomogenitäten innerhalb der DRG-Fallpauschalen bestehen. Weiterhin besteht häufig eine systematische regionale Arbeitsteilung mit Kliniken unterschiedlicher Schwerpunkte, die das Problem verschärft. Es war also geboten, den Versorgungsaufwand für onkologische Patienten systematisch zu evaluieren.

Im Frühjahr 2003 wurden aus einer Initiative der DGHO heraus deshalb zwei Projekte (eins zu den hämatologischen Neoplasien und eins zur internistischen Onkologie) ins Leben gerufen, die den Behandlungsaufwand in diesem Bereich analysieren sollten. Diese Projekte wurden gemeinsam von der Deutschen Krebsgesellschaft und der DRG-Research-Group in Münster getragen, mit inhaltlicher Unterstützung durch Mitglieder der DGHO. In einem weiteren Projekt, über das hier nicht berichtet wird, wurden durch die DAG-KBT ebenfalls mit Unterstützung der DRG-Research-Group die Knochenmark- und Stammzelltransplantationen evaluiert.

\begin{tabular}{ll}
\hline KARGER & ๑ 2005 S. Karger GmbH, Freiburg \\
Fax +49 7614520714 & Accessible online at: \\
$\begin{array}{l}\text { E-mail Information@Karger.de } \\
\text { www.karger.com }\end{array}$ & www.karger.com/onk
\end{tabular}




\section{Methodik}

Für einen begrenzten Zeitraum wurden alle relevanten Patienten lückenlos dokumentiert. Auf die gezielte longitudinale Analyse von selektierten «Problemfällen» wurde verzichtet, da sich Ergebnisse aus solchen Untersuchungen schlecht verallgemeinern lassen. Für das Projekt Hämatoonkologie wurden alle Patienten dokumentiert, die in den teilnehmenden Fachabteilungen behandelt wurden und eine hämatologische Neoplasie als Haupt- oder Nebendiagnose aufwiesen. Entsprechend wurden im Projekt internistische Onkologie alle Patienten mit soliden Tumoren als Haupt- oder Nebendiagnose dokumentiert. Onkologische Patienten in chirurgischen Abteilungen wurden nicht systematisch erfasst. Es wurden alle Patienten untersucht, die im dritten Quartal 2003 stationär in die Fachabteilungen der Projektteilnehmer aufgenommen wurden.

Für alle evaluierten Patienten wurden folgende Daten erfasst:

1) Datensatz gemäß $\S 21$ Krankenhausentgeltgesetz (KHEntgG), der auch an die Krankenkassen weitergeleitet und für die DRG-Gruppierung verwendet wird, und u. a. Diagnoseschlüssel nach dem ICD-10, Prozeduren nach dem OPS-301 sowie Angaben zur Dauer des Aufenthaltes enthält. Um eine möglichst korrekte und einheitliche Kodierung der Diagnosen und Prozeduren zu gewährleisten, wurde ein projekteigener Kodierleitfaden erstellt.

2) Klinische Informationen: zusätzlich wurden 8 vordefinierte klinische Eigenschaften dokumentiert. Therapieindikation (palliativ vs. kurativ), Therapiesituation (Primärtherapie, Rezidivtherapie, primär refraktäre Situation), schlechtester Allgemeinzustand während des stationären Aufenthaltes nach der WHO/ECOG Skala, Aufnahmegrund (geplant versus ungeplant), parenterales Morphin > 3 Tage (ja/nein), Neutropeniedauer in 5 Stufen zwischen 0 und $>20$ Tage, Patientenisolierung wegen multiresistenter Keime.

3) Patientenbezogene Dokumentation aufwendiger Maßnahmen: Diese Dokumentation beinhaltete 21 teure diagnostische Maßnahmen auch außerhalb des OPS-301, 97 teure Medikamente wie Zytostatika, Antibiotika usw. sowie Transfusionen und 84 übliche Zytostatikakombinationen. Über die Dokumentation der Zytostatika ließ sich neben den Zytostatikakosten nach Abschluss des Projektes auch die Intensität der Therapie ablesen.

$\mathrm{Zu}$ den Patienten-individuell dokumentierten Aufwandfaktoren wurde der Basisaufwand («Hotelkosten», stationäre Versorgung) hinzugerechnet. Hierbei wurde der Aufwand in «Aufwandsäquivalenten» berechnet und nicht die realen Kosten der einzelnen Kliniken berücksichtigt, da Aufwandsunterschiede untersucht werden sollten und nicht individuelle Kostenunterschiede, die auch von lokalen Kostenfaktoren und lokal ausgehandelten Preisen abhängen. Für Details der Methodik wird auf die Originalpublikationen verwiesen.

\section{Ergebnisse und Diskussion}

\section{Projektdurchführung}

40 Kliniken/Fachabteilungen nahmen an einem oder beiden Evaluationsprojekten teil, davon 23 universitäre Einrichtungen. Insgesamt wurden 24.271 Fälle dokumentiert, davon ca. 2/3 vollstationäre Fälle, 1/3 teilstationäre Fälle. Für die Auswertung wurden die Fälle nach dem Fallpauschalenkatalog des Jahres 2004 gruppiert. In einer ersten Auswertungsrunde wurden «klinischen Profile» für alle DRG erstellt. Hieraus konnten für jede DRG Kernparameter wie durchschnittliche Behandlungsdauer und durchschnittliche Aufwandsäquivalente abgelesen werden. Weiterhin konnte der Einfluss von Haupt- und Nebendiagnosen sowie Prozeduren auf den Be- handlungsaufwand innerhalb der DRG abgelesen werden. Im zweiten Schritt erfolgte die gezielte Analyse auf mögliche Inhomogenitäten, auch DRG-übergreifend. Aufgrund von Zwischenanalysen konnten zur Deadline Ende März 2004 erste Änderungsvorschläge beim InEK eingereicht werden. Im Nachfolgenden werden die wichtigsten Problemzonen aufgeführt, in denen sich in den Projektauswertungen Inhomogenitäten nachweisen ließen.

\section{Allgemeinzustand, Therapiesituation}

Es stellte sich heraus, dass ein schlechterer Allgemeinzustand während des stationären Aufenthaltes gemessen an der WHO/ECOG-Skala deutlich mit einem höheren Gesamtaufwand korreliert, ebenso wie die Notwendigkeit der parenteralen Gabe von Morphin-Präparaten. Weiterhin sind kurativ behandelte Patienten im Vergleich zu Patienten mit palliativem Therapieziel im Durchschnitt teurer. Dieser Unterschied ist ausgeprägter im Bereich der hämatologischen Neoplasien. Die Autoren waren trotz dieser Ergebnisse der Auffassung, dass sich diese Faktoren nicht für eine Anpassung des DRGSystems eignen, da sie entweder einem erheblichen Interpretationsspielraum unterliegen (Allgemeinzustand und The rapieindikation) oder zu Fehlanreizen führen können (i.v. Morphin).

Bei den akuten Leukämien war die Behandlung der Patienten in Remission im Durchschnitt um knapp die Hälfte aufwandsgeringer als die der restlichen Patienten. Der Remissionsstatus differenziert den Aufwand jedoch nicht ganz so gut wie Therapieintensität oder Komplikationen (s.u.) und ist weniger zuverlässig zu klassifizieren. Für 2005 wurde er vom InEK nicht berücksichtigt, weil die im Projekt offensichtlichen Aufwandsunterschiede in der bundesweiten Kalkulation - am ehesten durch eine ungenaue Dokumentation des Kriteriums «in Remission»- bisher nicht nachvollzogen werden konnten.

\section{Komplikationen und Nebendiagnosen}

Jeder praktisch tätige Onkologe wird erwarten, dass behandlungsbedürftige Komplikationen oder Nebendiagnosen zu längeren und aufwendigeren Aufenthalten führen. Die Definition der Nebendiagnose im jetzigen DRG System führt dazu, dass bereits wenig ausgeprägte und wenig kostenintensive Nebendiagnosen kodiert werden können und deswegen die PCCL-Matrix nur schlecht zwischen leichten und schweren Fällen diskriminiert. Es wurde deshalb mit den Projektdaten das Konzept «qualifizierter Nebendiagnosen» evaluiert. Hierfür wurden für klinisch relevante Interventionen, die häufig bei Komplikationen durchgeführt werden, «Komplikationspunkte» vergeben. Solche Komplikationspunkte wurden definiert für: i.v. Antibiotika ( $>3$ Tage ein Punkt, $>9$ Tage zwei Punkte), Transfusionen (TK ein Punkt, EK ein Punkt, $>5$ EK zwei Punkte), Pleurodese, Intervention im Gastrointestinalbereich (Stent, Blutstillung etc.). Bei Anwendung dieses einfachen Punktesystems fanden wir, dass die Anzahl der notwendigen Interventionen wesentlich besser mit dem Aufwand 


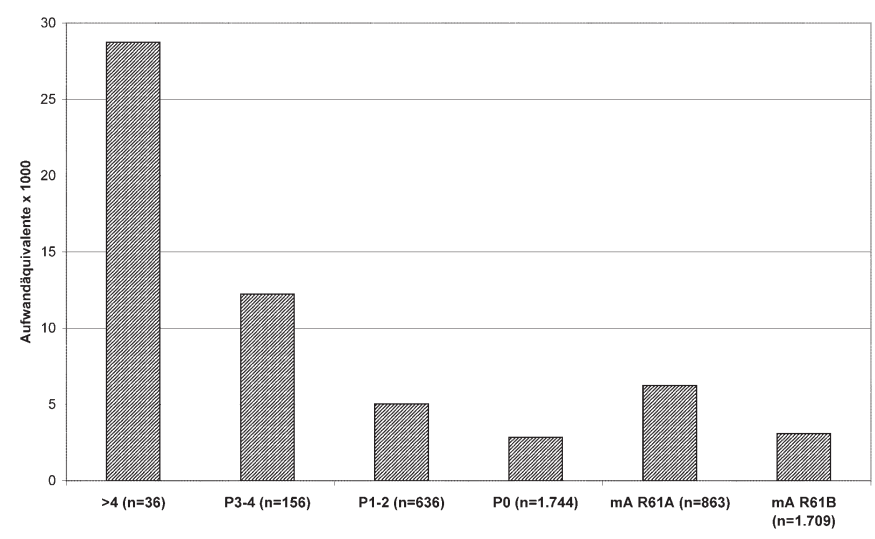

Abb. 1. Basis-DRG R61: Differenzierung des Aufwandes über die Anzahl der qualifizierten Nebendiagnosen/Prozeduren ( 0 bis $>4$ Punkte) im Vergleich G-DRG-Differenzierung über PCCL (R61A vs. R61B).

Tab. 1. Einfluss der Therapieintensität auf die DRG R60A (Akute Leukämien mit Chemotherapie)

\begin{tabular}{lllc}
\hline & Fallzahl & Verweildauer & Aufwand \\
\hline Keine intensive Chemotherapie & 371 & 21,0 & 9.873 \\
$\begin{array}{l}\text { Intensive «Induktions»- } \\
\quad \text { Chemotherapie }\end{array}$ & 158 & 32,1 & 18.337 \\
$\begin{array}{l}\text { Doppelzyklus Chemotherapie } \\
\text { (5) }\end{array}$ & 44,3 & 31.049 \\
\hline
\end{tabular}

korreliert als die Berechnung der Fallschwere mit der PCCLMatrix. Als Beispiel sei die Basis-DRG R61 Lymphom und nicht akute Leukämie in Abbildung 1 dargestellt. Das Konzept der qualifizierten, d.h. an Interventionen gekoppelten, Nebendiagnosen könnte die Abbildungsqualität in der Onkologie dementsprechend wesentlich verbessern. Für das Jahr 2005 wurde eine solche Änderung des Systems noch nicht systematisch eingeführt, durch die Berücksichtigung einzelner komplizierender Diagnosen und Prozeduren (z.B. Sepsis und Dialyse bei den Lymphomen R61) geht das InEK jedoch einen Schritt in diese Richtung.

\section{Intensität der Chemotherapie}

Die im Rahmen der Zusatzdokumentation erfassten Zytostatika wurden auch dazu benutzt, die Auswirkung der Intensität der Chemotherapie auf den Gesamtaufwand zu analysieren. Bei den akuten Leukämien (R60A) zeigte sich ein erheblicher Aufwandsunterschied abhängig von der Art der Chemotherapie (Tab. 1). Ähnlich großer Unterschiede fanden sich bei den Lymphomen und nicht-akuten Leukämien (R61), wenn zwischen intensiveren Therapien (typische Rezidiv-Chemotherapien), weniger intensiven Therapien (Standard-Therapien) und Patienten ohne Therapien differenziert wurde. Das InEK konnte die Intensität der Chemotherapie für das Jahr 2005 noch nicht berücksichtigen, da diese 2003 noch nicht im OPS301-Katalog erfasst wurde und demnach dem InEK 2004 für Berechnungen nicht zur Verfügung stand. 2004 wurde eine Differenzierung der Chemotherapieintensität erstmals in den OPS-301 aufgenommen. Das InEK sollte damit in der Lage sein, diese Analysen nachzuvollziehen und diesen wichtigen Diskriminator in das DRG-System aufzunehmen. Für eine optimale Diskriminierung wäre allerdings notwendig, die Definitionen der Chemotherapiestufen zu schärfen.

\section{Dauer der Neutropenie}

Bei den akuten Leukämien korrelierte die Dauer der Neutropenie in etwa gleichem Umfang mit dem Gesamtaufwand wie die Therapieintensität. Die Neutropeniedauer kann derzeit nicht differenziert im ICD-10 erfasst werden, nur eine Differenzierung über oder unter 20 Tage ist möglich. Bei den akuten Leukämien ist allerdings davon auszugehen, dass die Differenzierung nach Neutropeniedauer weitgehend die gleichen Patienten identifiziert wie die Differenzierung nach Chemotherapieintensität.

\section{Bildgebung/Staging}

Patienten, bei denen umfangreiche Staging-Untersuchungen und Bildgebung durchgeführt wurden, verursachten einen wesentlich höheren Gesamtaufwand als Patienten ohne diese Untersuchungen. Als Beispiel sei eine gemeinsame Auswertung von R61 Lymphom und nicht akute Leukämie und R62 Andere hämatologische und solide Neubildungen aufgeführt: umfangreiches Staging - 7.394 Aufwandsäquivalente, einfaches Staging - 4.107 Aufwandsäquivalente, kein Staging 2.338 Aufwandsäquivalente. Für 2005 fanden Staging-Untersuchungen keinen Eingang ins DRG System.

\section{Inhomogenitäten durch unterschiedliche Hauptdiagnosen}

Innerhalb der akuten Leukämien (R60) verursachte die Behandlung der myeloischen Leukämien einen höheren Aufwand als die Behandlung der lymphatischen Leukämien. Dies führte für 2005 zur Schaffung einer separaten Fallgruppe R63 Andere akute Leukämie.

Innerhalb der Lymphome und nicht akuten Leukämien (R60) benötigten sowohl die Burkitt-Lymphome als auch diejenigen myelodysplastischen Lymphome, die mit Chemotherapie behandelt wurden, einen deutlich erhöhten Aufwand. Dieses Problem könnte entweder durch einen Splitt auf Basis der Diagnosen oder aber durch einen Splitt auf Basis der Therapieintensität behoben werden. Dies ist für 2005 noch nicht erfolgt.

Vergleichsweise geringe Inhomogenitäten ergaben sich für unterschiedliche Hauptdiagnosen innerhalb der einzelnen Fallgruppen in der Onkologie. So war innerhalb von G60 der Aufwand für Malignome des unteren Verdauungstraktes höher als für Malignome des oberen Verdauungstraktes. Dies wurde vom InEK für 2005 nicht berücksichtigt. Innerhalb von H61 (Malignome an hepatobiliärem System und Pankreas) war in den Projektdaten der Aufwand für Malignome des Pankreas überdurchschnittlich hoch. Das InEK führte aufgrund der offiziellen Kalkulationsdaten eine Anpassung durch, die zu einer relativen Aufwertung von Pankreasschwanzkarzinomen und einigen anderen Tumoren führt. 
Abb. 2. Mehrtagesfälle der Basis-DRGs E71, G60, H61, I65 und J62: Varianz der Aufwandäquivalente des Gesamtaufwandes (Gesamt) und des Gesamtaufwandes abzüglich des Zytostatika aufwandes (G-Z) bei Fällen mit einer Verweildauer (VWD) von 2-5 Tagen. Es wird deutlich, dass ein erheblicher Anteil der Varianz durch die Zytostatika verursacht wird.

\section{Aufwand für teure Medikamente}

Es konnte sowohl bei hämatologischen als auch onkologischen Patienten nachgewiesen werden, dass eine erhebliche Varianz des Aufwandes durch einzelne, sehr teure Medikamente verursacht wird. Dies fällt insbesondere bei kurz dauernden, medizinisch ansonsten relativ unkomplizierten Behandlungen ins Gewicht (Abb. 2). Dieses Ergebnis war vorhersehbar und ist leicht nachzuvollziehen, wenn man z.B. die Behandlungskosten für eine Therapie mit Trastuzumab/Paclitaxel einer Therapie mit Doxorubicin/Cyclophophamid beim Mammakarzinom gegenüberstellt. Aufgrund dieser erheblichen Kostenunterschiede wurde dem InEK vorgeschlagen, dieses Problem durch Zusatzentgelte (ZE) für einzelne teure Medikamente zu lösen. Dieser Vorschlag wurde inzwischen als wesentliche Neuerung für 2005 umgesetzt. Dabei wurden untere Schwellenwerte eingeführt, die teilweise deutlich über den Standardtherapiedosen liegen, nämlich bei solchen Medikamenten, bei denen die Standardtherapie nicht kostenauffällig war. Dies geschieht mit der Überlegung, dass ZE einen Risikoschutz bei Kostenausreißern darstellen und deshalb nicht jede Gabe der ZE-Medikamente zusätzlich vergütet werden muss. Die Schwellenwerte bergen trotzdem ein gewisses Risiko von Fehlanreizen.

\section{Weitere Probleme}

Im Rahmen der Projektauswertung zeigte sich, dass Tumorpatienten in nicht-onkologischen Fallgruppen (d.h. Tumorerkrankung nur Nebendiagnose), insbesondere im Bereich der Infektionskrankheiten, ein deutlich von der InEK Stichprobe abweichendes Kollektiv darstellen und vermutlich aufwändiger zu behandeln sind. Dies konnte im Rahmen des Projektes nicht erschöpfend bearbeitet werden, da keine nicht-onkologischen Vergleichspatienten untersucht wurden. Durch das InEK wurde für 2005 kein Splitt vorgenommen.

Bei einzelnen Diagnosen ließ sich aus den Projektdaten eine offensichtlich heterogene Kodierung von Zentrum zu Zentrum ablesen, obwohl als Hilfe ein Projekt-Kodierleitfaden zur Verfügung stand. Weiterhin war ein Teil der Datenerfassung (z.B. zusätzliche Charakteristika wie Neutropeniedauer, Therapieindikation) unvollständig. Dies zeigt, dass für ein funktionierendes System alle Definitionen in den Klassifikationssystemen und Kodierrichtlinien eindeutig, einfach und konkordant zur klinischen Praxis zu halten sind und nur ausreichend valide Kodes für die Einteilung der Fallgruppen verwendet werden dürfen. Die Fachgesellschaften stehen in Kontakt mit dem DIMDI und hoffen auch in diesem Bereich auf Verbesserungen in den nächsten Jahren.

\section{Zusammenfassung und Ausblick}

Durch den großen Einsatz der teilnehmenden Kliniken konnten umfangreiche Daten generiert werden, aus denen wir wichtige Erkenntnisse zum Behandlungsaufwand bei onkologischen Patienten ziehen können. Diese Daten (und ähnliche Daten aus anderen Projekten) führten dazu, dass als bedeutende Neuerung für die Onkologie ab 2005 Zusatzentgelte für teure Medikamente eingeführt wurden. Als wichtigste weitere Aufwandsfaktoren konnten die bei den Lymphomen und Leukämien die Therapieintensität und über alle Diagnosen hinweg interventionspflichtige Komplikationen nachgewiesen werden. Die Autoren hoffen, dass diese Faktoren für 2006 in das System integriert werden können. Auch in zahlreichen weiteren Aspekten besteht noch Anpassungsbedarf. Die Änderungen für 2005 beweisen, dass das System grundsätzlich anpassungsfähig ist und die Autoren ermutigen jeden interessierten Kollegen, sich aktiv in die DRG-Arbeitsgruppen der Fachgesellschaften einzubringen.

Weitere Informationen zum Projekt finden Sie unter: http:// smc-inet3.uni-muenster.de/drg/onko/

Informationen zum Arbeitskreis der DGHO: http://www.dgho. del_cache/cms_19.html

\section{Liste der teilnehmenden Kliniken/Fachabteilungen}

Charité, Campus Virchow-Klinikum, Medizinische Klinik mit Schwerpunkt Hämatologie u. Onkologie, Berlin; Charité, Robert-Rössle-Klinik, Klinik für Chirurgie u. chirurgische Onkologie, Berlin; Städtisches Klinikum Braunschweig, Klinik für Hämatologie u. Onkologie, Braunschweig; Klinikum Bremen Mitte, Klinik Innere Medizin I, Bremen; Klinikum Bremen Mitte, Klinik Innere Medizin II, Bremen; HUMAINE Klinik Dresden, Klinik für Innere Medizin, Dresden; Universitätsklinikum Carl Gustav Carus, Medizinische Klinik u. Poliklinik 1, Dresden; Universitätsklinikum Düsseldorf, Klinik für Kinder-Onkologie,, Düsseldorf; UniversitätsFrauenklinik Erlangen, Erlangen; Universitätsklinikum Essen, Klinik u. Poliklinik für Innere Medizin (Tumorforschung), Essen; Krankenhaus Nordwest, Medizinische Klinik II, Frankfurt; Universitätsklinikum Freiburg, Medizinische Klinik u. Poliklinik I, Freiburg; Universitätsklinikum Freiburg, Medizinische Klinik u. Poliklinik II, Freiburg; St. Marien-Hospital, Klinik für Hämatologie u. Onkologie, Hagen; Universitätsklinikum Hamburg-Eppendorf, Einrichtung für Knochenmarktransplantation der Abt. Onkologie-Hämatologie, Hamburg; Universitätsklinikum HamburgEppendorf, Zentrum für Innere Medizin, Medizinische Klinik II, Ham- 
burg; Medizinische Hochschule Hannover, Abteilung Hämatologie Hämostaseologie u. Onkologie, Hannover; Medizinische Hochschule Hannover, Abteilung für Gastroenterologie , Hannover; Universitätsklinik Heidelberg, Medizinische Klinik u. Poliklinik V, Heidelberg; Klinik für Knochenmarktransplantationen u. Hämatologie/Onkologie, Idar-Oberstein; Städtisches Krankenhaus Kiel, 2. Medizinische Klinik, Kiel; Universitätsklinikum Leipzig, Medizinische Klinik II, Leipzig; Universitätsklinikum Mainz, I. Medizinische Klinik, Mainz; Universitätsklinikum Mainz, III. Medizinische Klinik, Mainz; Klinikum Minden, Klinik für Internistische Onkologie u. Hämatologie, Minden; St. Marien-Hospital, I. Medizinische Klinik, Mülheim; Klinikum der Universität München - Großhadern, Medizinische Klinik III, München; Universitätsklinikum Münster, Medizinische Klinik u. Poliklinik A, Münster; Universitätsklinikum Münster, Klinik u. Poliklinik für Kinderheilkunde, pädiatrische Hämatologie/Onkologie, Münster; Universitätsklinikum Münster, Klinik u. Poliklinik für Frauenheilkunde u. Geburtshilfe, Münster; Klinikum Nürnberg, Medizinische Klinik 5, Nürnberg; Humaine Schloßbergklinik Oberstaufen, Oberstaufen; Klinikum Offenbach, Chirurgische Klinik I, Offenbach; Klinikum Oldenburg, Klinik für Onkologie u. Hämatologie, Oldenburg; Klinikum der Universität Regensburg, Abt. f. Hämatologie u. Internistische Onkologie, Regensburg; Klinikum der Universität Regensburg, Gastroenterologie, Regensburg; Caritasklinik St. Theresia, Klinik für Hämatologie u. Onkologie, Saarbrücken; Caritasklinik St. Theresia, Frauenklinik mit Neonatologie, Saarbrücken; Universitätsklinik Tübingen, Medizinische Klinik, Abt. II, Tübingen; HSK Dr.-Horst-Schmidt-Kliniken, Innere Medizin III, Wiesbaden. 